\title{
Acquiescence and Person Differential Functioning: Solving Person Differential Functioning with Balanced Scales
}

\begin{abstract}
Ricardo Primi (Universidade São Francisco, and EduLab21, Ayrton Senna Institute, Brazil), Daniel Santos (Universidade de São Paulo and EduLab21, Ayrton Senna Institute, Brazil), Filip De Fruyt, (Ghent University, Belgium and EduLab21, Ayrton Senna Institute, Brazil) \& Oliver P. John (University of California, Berkeley and EduLab21, Ayrton Senna Institute, Brazil),
\end{abstract}

Draft version 2.0 of 8/8/2018. This paper has not been peer reviewed. Please do not copy or cite without author's permission.

\footnotetext{
Author Note

Ricardo Primi, Post Graduate Program in Psychology, Universidade São Francisco. Campinas, Brazil; Filip De Fruyt, Department of Developmental, Personality and Social Psychology, Ghent University, Gent, Belgium; Oliver P. John, Department of Psychology and Institute of Personality and Social Psychology, University of California, Berkeley, United States; Daniel Santos, Faculty of Economics, Administration and Accounting of Ribeirão Preto, University of São Paulo, Ribeirão Preto, Brazil.

This article is part of a research project supported by the Ayrton Senna Foundation. The first author also received a scholarship from the National Council on Scientific and Technological Development $(\mathrm{CNPq})$.

Correspondence should be addressed to: Ricardo Primi, Universidade São Francisco, Rua

Waldemar César da Silveira, 105, Campinas, São Paulo, CEP 13045-510; e-mail: rprimi@mac.com

Word count: 5044
} 


\begin{abstract}
Likert-type self-report scales are frequently used in large-scale educational assessment of socialemotional skills. Self-report scales rely on the assumption that their items elicit information only about the trait they are supposed to measure. However, different response biases may threat this assumption. Specifically in children, the response style of acquiescence is an important source of systematic error. Balanced scales have been proposed as a solution to control for acquiescence, but the reasons why this design feature worked from the perspective of modern psychometric models have been underexplored. Three methods for controlling for acquiescence are compared: (a) Classical ipsatization by partialling out the mean, (b) an Item Response Theory method to measure Person Differential Functioning (DPF), and (c) random intercept item factor analysis. Comparative analyses are conducted on simulated ratings and on self-ratings provided by 40,649 students (aged 11 to 18) on a fully balanced 30-item scale assessing Conscientious Selfmanagement. Acquiescence bias was explained as person differential item functioning and it was demonstrated that: (a) the acquiescence index is highly related to DPF, (b) balanced scales resolve DPF, and (c) that random intercept factors are highly related to DPF and the substantive factor is controlled for DPF.
\end{abstract}

Keywords: response styles, personality assessment, item response theory, random intercept model 


\section{Acquiescence and Person Differential Functioning: Solving Person Differential Functioning with Balanced Scales}

Likert-type self-report scales are increasingly used in large-scale assessments of socio emotional skills in primary and secondary education (Abrahams et al., in press). Kyllonen, Lipnevich, Burrus and Roberts (2014) reviewed constructs and methods of social-emotional skill assessment and recommended the use of self-reports because they are uncomplicated, straightforward to develop, and easy to administer in large-scale applications.

A pertaining issue, however, with the use of self-report Likert-scales is how to deal with response biases. Response bias refers to systematic factors that affect responses but are not related to the construct of interest. Since self-report depends on complex cognitive factors such as self-knowledge, memory, reading capability and motivation to disclose information about oneself it is highly sensitive to response bias. Well-known forms of response biases are: (a) acquiescence: i.e. the tendency to choose responses stating agreement regardless of the content of the item, (b) disacquiescence: i.e. the tendency to choose responses stating disagreement regardless of the content of the item, (c) extreme response bias: i.e. the tendency to use the end points of a scale regardless of the content, (d) middle response bias: i.e. the tendency to use the midpoint of the Likert-scale regardless of the content, (e) social desirability bias: i.e. the tendency to answer questions in a way to present oneself in a positive way, and (f) group reference bias: i.e. the systematic differences across respondents regarding internalized group/culture frames of reference to make relative judgments about themselves (e.g., Duckworth \& Yeager, 2015; He, Bartram, Inceoglu, \& van de Vijver, 2014; Wetzel \& Carstensen, 2015). McCrae, (2018) have shown that these influences, named method variance, accounts for $40 \%$ of the variance in self reports. 
This paper will focus on acquiescence bias, and investigate how different methods to correct such bias operate and are equivalent. Acquiescence was chosen given its pronounced effects, especially in self-reports of students relative to adults. Soto, John, Gosling, and Potter (2008) have convincingly shown that acquiescence variance is highest for ages 10 to17, and then declining to age 18 equaling adult levels. Acquiescence demonstrated to influence the internal structure of assessment instruments and their criterion validity (Rammstedt, Goldberg \& Borg, 2010; Primi, De Fruyt, John, \& Santos, 2018). It is very stable influence over a period of eight years as was shown by Wetzel, Lüdtke, Zettler, and Böhnke (2016). Therefore, this bias needs to be dealt with in student assessments and thoroughly understood.

\section{Acquiescence: Measurement and correction}

Acquiescence refers to the tendency to agree with questions regardless of the content of the item. One way to examine acquiescence is to include positively-keyed (PK) and negativelykeyed items (NK), that is, markers of opposite poles of a trait. Imagine an item designed to measure negative emotional regulation, such as $i+$ : 'I adapt easily to new situations without worrying too much', with students asked to respond on a scale with '1' (not at all like me), '2' (little like me), '3' (moderately like me), '4' (a lot like me) and '5' (completely like me). Also, an antonym pair item is included such as $i$-: 'I have trouble controlling my anxiety in difficult situations'. A person very high in acquiescence will tend to respond with ' 4 ' or ' 5 ' to both items. A person with low acquiescence, that is, one that responds more to the item's psychological meaning and who is higher on emotional regulation will tend to give reflected responses, for example ' 4 ' to $i+$ and ' 2 ' to $i$-.

Soto et al. (2008) computed acquiescence in the Big Five Inventory by calculating for each individual $j$ a mean on 16 item pairs representing opposite poles of big five traits (index of 
acquiescence $a c q_{j}$ ). Since these items measure the same trait but come from opposite ends of the trait continuum, agreement with positive items should co-occur with disagreement with negative items. Therefore, the expected score on this index will be $a c q_{j}=3$. If $a c q_{j}>3$ or $a c q_{j}<3$ it will indicate high acquiescence or disacquiescence respectively. Then test items responses $y_{i j}$ of individual $j$ to the $i$ items of the test can be transformed using the formula: $r_{i j}=\left(y_{i j}-a c q_{j}\right)$. This is done to partial out the effect of acquiescence on all the individual's responses.

In case we have fully balanced scales where the number of pairs is equal to the number of items divided by two: $n_{i} / 2$, this transformation will produce a set of ipsative variables $r_{i j}$. That is, $a c q_{j}$ will be the mean of agreement on all items before reversing negative items. Therefore, the sum of transformed items $r_{i j}$ for each individual $j$ will be equal to zero. This could look like we have removed all between-subject variance and are left with only within-subject variance (Chan \& Bentler, 1998).

Back in 1999, Ten Berge noted that an ipsative transformation of balanced personality scales containing positive and negative items is a special case with peculiar properties. Usually when all items correlate positively with each other, like items of a cognitive test, ipsatization removes the first principal component that has substantive meaning, that is, a general factor. This is usually not what researchers want to achieve. But in a balanced personality scale with carefully selected items as psychological antonym pairs, that is, reflecting opposite poles of an underlying single dimension, ipsatization removes response bias.

This occurs because in such a case substantive trait information will be located in the negative covariances between positive and negative items as well as in the positive covariance within positive items and negative items. Any positive covariance between positive and negative items will indicate non-substantive variance (systematic construct irrelevant variance). 
Consistently agreeing or disagreeing with opposite pairs may reflect answering style differences, incomprehension, low motivation, confirmation bias, or careless responding (Garrido, Golino, Nieto, Peña, \& Molina, 2018). Therefore, when ipsatizing, only this globally positive manifold variance reflecting bias is removed from the covariance matrix. But since the main trait information is within contrasted positive and negative covariances, substantive information is not really removed (Ten Berge, 1999). This is a clever way to identify and isolate substantive trait information from bias or construct irrelevant variance.

In balanced scales, the ipsative transformation is not really a full ispsative transformation because after reversing negative ipsatized variables and summing all variables $r_{i j}$ for an individual $j$, they don't sum to a constant across individuals. There will be still between-subject variance left, because the ipsatization occurs only with a parcel of $y_{i j}$ variance related to the positive manifold across positive and negative items.

The way noise-canceling headphones work, provides a good analogy of what is going on using this correction. These headphones have a microphone inside their cups to "hear" external noises and neutralize their distorting effects. These headsets' electronics create a new inverted version of the noise sound wave (180 degrees out of phase) that is send into a speaker. In the speaker, you hear now signal, noise and inverted noise waves. Since there are two noise waves, one positive and one negative, they cancel each other out and a clearer signal remains.

This is analogous to what happens with balanced personality scales: Trait is signal, bias is noise. The only difference is that signal, not noise, is inverted - via positive and negative items and inserted in the scale. Noise, that is, acquiescence is a positive signal that happens to occur on both positive and negative items. When scoring the test, negative items are reversed, and item scores are summed into a scale score. While doing this, negative items are inverted aligning them 
to the positive pole, transforming the signal in the same "phase" as positive items, finally ending up with equal amounts of positive and negative noise that cancel each other out. In synthesis, if we carefully develop balanced items to isolate bias in the positive manifold, the remaining variance after ipsatizing will be a purified measure.

\section{Acquiescence as person differential functioning (DPF)}

Johanson and Osborn (2004) proposed a new operational definition of acquiescence as differential person functioning (DPF). Differential Item functioning (DIF) occurs when subjects with the same level on the construct, but from different groups, have different probabilities of endorsing a particular answer, for instance agree with a description. If an item measures mainly one dimension and there are two persons with the same level on this dimension they should have the same response on the item. Any difference in their responses will be treated as DIF. In the upper part of Figure 1 there is a representation of a data matrix where columns are items parametrized by their index of difficulty $b_{i}$ and rows are persons from two groups $\mathrm{g} 1 / \mathrm{g} 2$ parametrized by their theta level $\theta_{j}$. Usually, DIF assesses if items have invariant properties for persons with the same level on the construct but from different groups (gender, race, etc.). If they don't, it could potentially bias group comparisons, making meaningful conclusions problematic. One way to solve DIF is to model group-by-item difficulty interactions estimating different item difficulty per group on items exhibiting DIF (represented in this figure as $b_{i g}$ ). When not accounted for, these differences will be confounded with latent scores, making the groups appear more different than they really are on the dimension of interest.

Johanson and Osborn (2004) proposed that one can also look at groups of items instead of persons. For instance, in balanced scales where we have groups of positive and negative items (represented in the lower part of Figure $1 \mathrm{~g} 1$ and g2), two items can measure the same dimension 
of responsibility (a facet of conscientiousness) - for instance: $i+$ : 'I only make promises I know I will be able to fulfill' versus $i$ - 'I sometimes don't keep the promises I've made' - and have the same level of difficulty. When an individual answers ' 5 ' on an item $i+$ on a five point Likertscale, he or she should give a ' 1 ' to the item $i$-. When the negative response is rescored, both will be ' 5 '. If responses to these items happen to be different, we are seeing Differential Person Functioning (DPF), that is, a person differently reacts to two types of items that measure the same trait with equivalent difficulty but differ in some item feature.

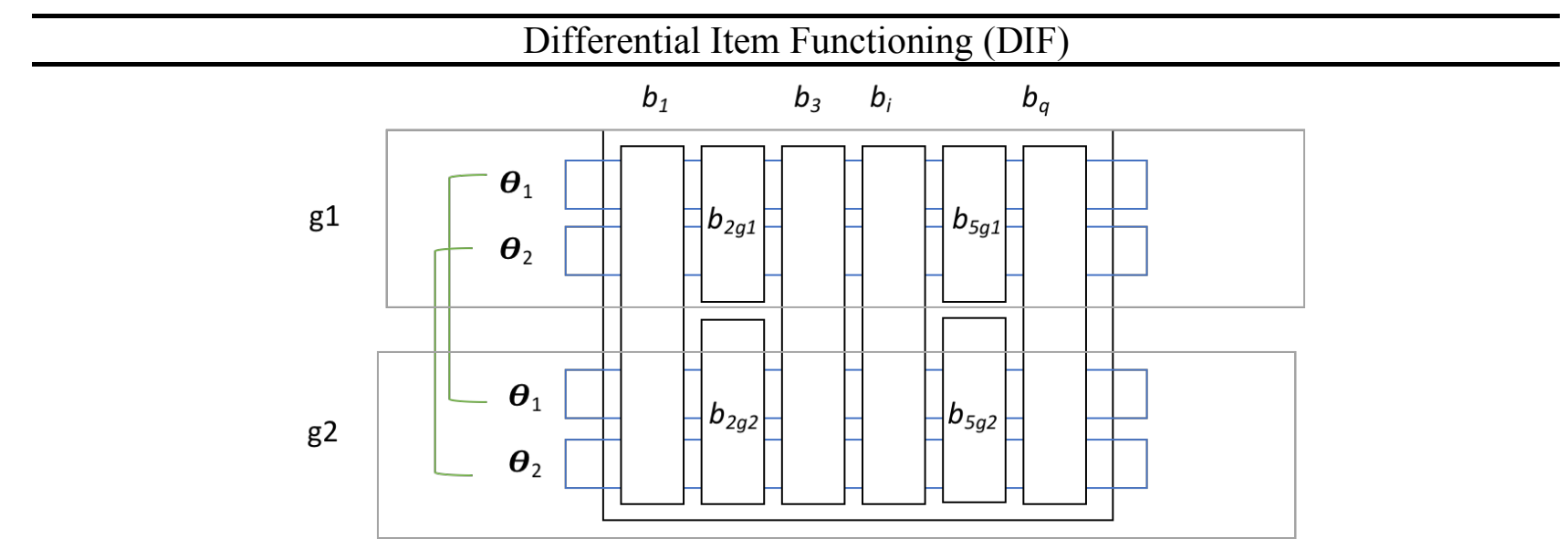

Differential Person Functioning (DPF) 


\section{Differential Item Functioning (DIF)}

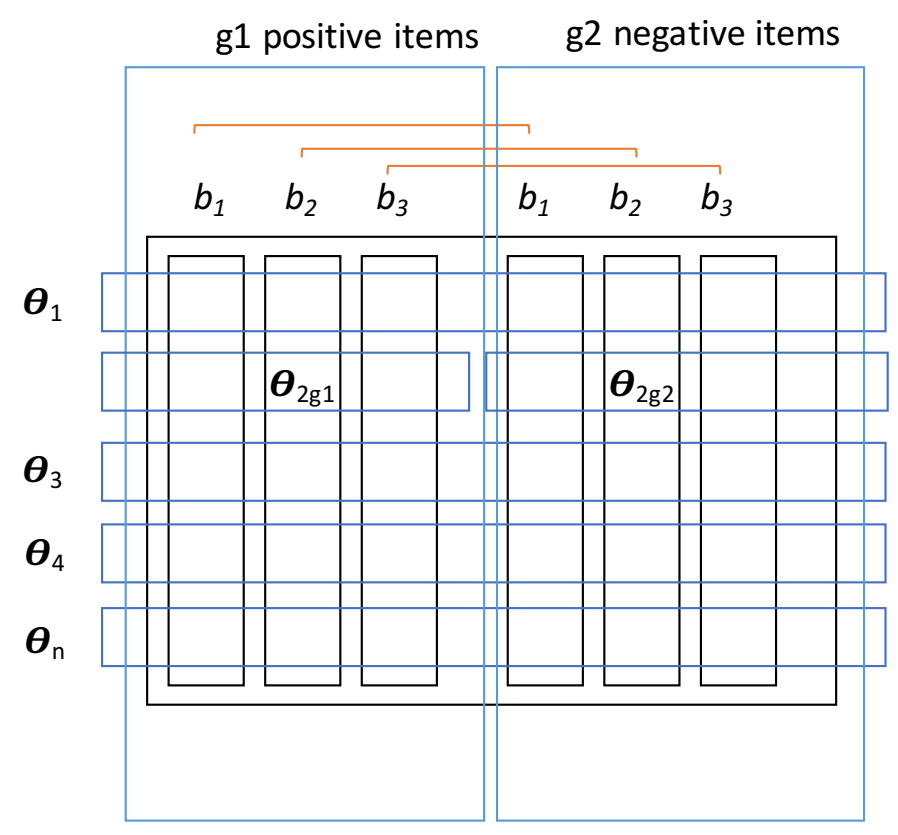

Figure 1. Representations of parametrization of Differential Item Functioning (upper half) and Differential Person Functioning (lower half).

This operationalization makes it possible to conceptualize acquiescence bias, as well as other forms of response styles (see for instance: Mirowsky \& Ross, 1991), as DPF and treat them using Item Response Theory (IRT) methods. This is coherent with the definition of bias as an additional dimension of individual differences pertaining to persons. Therefore, it needs to be modeled as a multidimensional IRT. One advantage of this method is to measure response styles' dimensions together with substantive traits.

\section{Acquiescence control as a method of solving DPF}

While solving DIF is relatively straightforward, solving DPF is more challenging. DIF is solved by splitting the items by groups of interest, estimating different item parameters for each group. Since we are interested in person parameters this makes sense because it disentangles group differences of non-target dimensions from person parameters. In case of DPF it doesn't 
make sense, for instance, to estimate two latent traits, i.e. one based on positive and another on negative items, when we have substantive evidence that this trait is unidimensional. So how can DPF be solved?

We argue that Ten Berge's (1999) ipsatizing method is a way to solve DPF. What happens when scores are recoded partialling out the mean (acquiescence)? Consider a six-item scale composed of three pairs of antonym items scored on a 5-point Likert scale. Let $i=1,2,3$ be the positive, and $i=a, b, c$ be the respective negative items, and $x_{i j}$ be the original response of subject $j$ on item $i$. The aquiescence index $a c q_{j}$ of a subject $j$ is given by:

$$
\operatorname{acq}_{j}=\frac{1}{6}\left[\sum_{i=1}^{3} x_{i j}+\sum_{i=a}^{c} x_{i j}\right]
$$

The average agreement score (classical test score) $s c r_{j}$ of a subject $j$ is given by:

$$
\operatorname{scr}_{j}=\frac{1}{6}\left[\sum_{i=1}^{3} x_{i j}+\sum_{i=a}^{c}\left(6-x_{i j}\right)\right]
$$

And the recoded score:

$$
s c r . r e c_{j}=\frac{1}{6}\left[\sum_{i=1}^{3}\left(x_{i j}-a c q_{j}\right)-1\left(\sum_{i=a}^{c}\left(x_{i j}-a c q_{j}\right)\right)\right]
$$

That simplifies to ${ }^{1}$ :

${ }^{1}$ This formula makes the analogy with noise canceling headphones more salient. In headphones you have audio signal $+(+$ noise $)+(-$ reflected noise $)$ therefore, noise cancel out. In balanced scales: a) positive items are composed of +trait signal $+(+$ acquiescence bias $)$ and b) negative items are composed of: -trait signal $+(+$ acquiescence bias $)$. When we compute $[+$ trait signal + (+acquiescence bias)] - [-trait signal + (+acquiescence bias $)]$ we end up only with trait signal and acquiescence cancels out. 


$$
\operatorname{scr} \cdot \operatorname{rec}_{j}=\frac{1}{6}\left[\sum_{i=1}^{3} x_{i j}-\sum_{i=a}^{c} x_{i j}\right]
$$

It is also interesting to mention that for balanced scales the original scores are a linear transformation of original scores $s c r_{j}=\operatorname{scr} \cdot r e c_{j}+3$, that is, the classic scores are already automatically recoded and have acquiescence partialled out ${ }^{2}$.

The simplified formulation of recoded scores $s c r . r e c_{j}$ can be used to understand how ipsatization solves DPF. Table 1 shows eight response patterns on six items as exemplified above and their corresponding: average $\left(s c r_{j}\right)$, acquiescence $\left(a c q_{j}\right)$ and recoded $\left(s c r . r e c_{j}\right)$ scores. Assume that each item pair $(i 1 / i a, i 2 / i b, i 3 / i c)$ has the same level of difficulty. These patterns vary in terms of trait and acquiescence.

Patterns P1 and P2 show the maximum and minimum scores that are possible when an individual is maximally consistent, that is, with no acquiescence (no DPF). Recoded scores scr. $r e c_{j}$ vary from -2 to 2 . Patterns $\mathrm{P} 3$ and $\mathrm{P} 4$ also show maximum and minimum possible scores, but now when a person is maximally inconsistent, that is, with acquiescence or disacquiescence maximized (presence of DPF). Now recoded scores $s c r . r e c_{j}$ are 0.

Table 1. Eight response patterns on six items: Three PK (il, i2,i3) and three NK (ia, ib, ic) and their corresponding average $\left(s c r_{j}\right)$, acquiescence $\left(a c q_{j}\right)$ and recoded $\left(s c r . r e c_{j}\right)$ scores.

${ }^{2}$ If $s c r_{j}=\frac{1}{6}\left[\sum_{i=1}^{3} x_{i j}+\sum_{i=a}^{c}\left(6-x_{i j}\right)\right]$ and scr.rec $c_{j}=\frac{1}{6}\left[\sum_{i=1}^{3} x_{i j}-\sum_{i=a}^{c} x_{i j}\right]$ then $\frac{1}{6} \sum_{i=1}^{3} x_{i j}+\frac{1}{6} \sum_{i=a}^{c}\left(6-x_{i j}\right)=\frac{1}{6} \sum_{i=1}^{3} x_{i j}-\frac{1}{6} \sum_{i=a}^{c} x_{i j} \Rightarrow \frac{1}{6} \sum_{i=1}^{3} x_{i j}+\frac{1}{6} \sum_{i=a}^{c} x_{i j}+3=$ $\frac{1}{6} \sum_{i=1}^{3} x_{i j}-\frac{1}{6} \sum_{i=a}^{c} x_{i j}$ 


\begin{tabular}{|c|c|c|c|c|c|c|c|c|c|c|c|}
\hline $\begin{array}{l}\text { Resp. } \\
\text { pattern. }\end{array}$ & Trait level & $\begin{array}{l}\text { Aquiescence } \\
\text { /Inconsistency }\end{array}$ & $i 1$ & $i 2$ & $i 3$ & $i a$ & $i b$ & $i c$ & $s c r_{j}$ & $a c q_{j}$ & scr.rec $_{j}$ \\
\hline $\mathrm{P} 1$ & Max & Min & 5 & 5 & 5 & 1 & 1 & 1 & 5.0 & 3 & 2.0 \\
\hline $\mathrm{P} 2$ & Min & Min & 1 & 1 & 1 & 5 & 5 & 5 & 1.0 & 3 & -2.0 \\
\hline P3 & Max & Max & 5 & 5 & 5 & 5 & 5 & 5 & 3.0 & 5 & 0.0 \\
\hline $\mathrm{P} 4$ & Min & Max & 1 & 1 & 1 & 1 & 1 & 1 & 3.0 & 1 & 0.0 \\
\hline P5 & High & Low & 5 & 4 & 4 & 1 & 2 & 2 & 4.3 & 3 & 1.3 \\
\hline P6 & Low & Low & 1 & 2 & 2 & 5 & 4 & 4 & 1.7 & 3 & -1.3 \\
\hline P7 & High & $\begin{array}{l}\text { Moderate } \\
\text { high } \\
\text { Moderate }\end{array}$ & 5 & 4 & 4 & 2 & 3 & 3 & 3.8 & 3.5 & 0.8 \\
\hline $\mathrm{P} 8$ & Low & high & 2 & 3 & 3 & 5 & 4 & 4 & 2.2 & 3.5 & -0.8 \\
\hline
\end{tabular}

Individuals with patterns P5 and P7 are high on the trait and have the same answers on positive items, but P5 has low acquiescence, that is, no DPF, and P7 high acquiescence, relatively high DPF. Here we can see more clearly DPF phenomena. The expected response on negative items of an individual with responses ' 5 ', ' 4 ', ' 4 ' on the three positive items are ' 1 ', ' 2 ', '2'. P7 instead had answered ' 2 ', ' 3, ' 3 ', that is, he/she did not answer in the same way when confronted with questions measuring the same trait with the same difficulty, but phrased negatively. Therefore, for this individual, it is relatively easy to agree because they agree to anything. As a consequence, scores on positive items are relatively higher for an acquiescent individual than for a non-acquiescent individual. The DPF correction then lowers down P7's score. The recoded score is .8 for P7 as compared to 1.3 for P5. Examples P6 and P8 have the same logic but now on the low end. The correction now makes scores to be less negatively extreme because it takes into account acquiescence.

These examples illustrate how DPF is solved. The amplitude of recoded scores were reduced from $2.6(-1.3-1.3)$ to $1.6(-.8-.8)$. Since part of the agreement is explained by acquiescence the recoding makes them less extreme. In this way acquiescence variance is 
partialled out, keeping the remaining variance that is consistent. Any variance related to acquiescence is removed. In the extreme cases with maximum acquiescence, scores will be in the middle with no variance (examples P3 and P4). This method is making scores less extreme as a function of individual differences on acquiescence index. It is interesting to note that all these changes happen silently in balanced scales ${ }^{3}$.

\section{Item factor analysis approach that corrects DPF}

Savalei and Falk (2014) revised three statistical models to analyze balanced scales: a factor model for ipsatized variables of Chan and Bentler (1998), an extension of Ten Berge (1999) using exploratory factor analysis with the target rotation proposed by Ferrando, LorenzoSeva, and Chico (2003), and a confirmatory factor analytic approach detailed by MaydeuOlivares and Coffman, (2006) called random-intercept factor analysis. Although the three approaches performed very well, the random-intercept approach performed best. This was also the case for a simulation study conducted by Garrido et al. (2018).

In unidimensional scales the random-intercept model can be written as:

$$
x_{i j}=\left(\mu_{i}+\zeta_{j}\right)+\lambda_{i} f_{j}+e_{i j}
$$

Where $x_{i j}$ is the response of subject $j$ on item $i, \mu_{i}$ is the general item $i$ intercept (reverse of item difficulty), $\zeta_{j}$ is the random intercept of individual $j, \lambda_{i}$ is the factor loading of item $i$ on

${ }^{3}$ There is an alternative method of recoding responses that divides $x_{i j}-a c q_{j}$ by the within person standard deviation of responses $S D_{j}$ on item responses. Then recoded scores calculated by: scr.z.rec $c_{j}=\frac{1}{6}\left[\sum_{i=1}^{3}\left[\left(x_{i j}-a c q_{j}\right) / S D_{j}\right]-1\left[\left(x_{i j}-a c q_{j}\right) / S D_{j}\right]\right]$. When doing the amplitude of the scores will be similar for acquiescence individuals as compared to non-acquiescence individuals. Since $S D_{j}$ is dependent on $a c q_{j}$ this transformation may undo part of the DPF correction (see https://github.com/rprimi/acqu mirt for more details on that). 
latent factor $f, f_{j}$ is the score of individual $j$ on the latent factor and $e_{i j}$ is the error of prediction of the response of individual $j$ on item $i$. Note that item difficulty is split in two components: (a) general item difficulty $\mu_{i}$ that varies between items but is the same for all individuals, and (b) the random intercept $\zeta_{j}$ that varies between individuals but is the same for all the items that the individual answers. Random intercept is estimated fixing a loading of 1 in all items before reversing. This model also assumes that random intercept is uncorrelated with $e_{i j}$ and $f_{j}$. Note that in balanced scales random intercept $\zeta_{j}$ will capture positive covariance between items being therefore an estimate of acquiescence similar to the simple $a c q_{j}$ score. The final estimate of latent factor $f_{j}$ have acquiescence partialled out. Therefore $f_{j}$ is similar to $s c r . r e c_{j}$. Consequently, this is also a solution to DPF.

\section{Item Response Theory approach that corrects DPF}

Since factor analytic models can be transformed in IRT by performing an alternative parametrization (Kamata \& Bauer, 2008, Takane, \& Leeuw, 1987) it is possible to use random intercept as a starting point of an IRT model for the correction for acquiescence and link this model with the DPF correction. The random intercept model can be estimated using a multidimensional version of the graded response model (Chalmers, 2012, Samejima, 1968). For $k$ likert options the graded response model recodes responses into $t=k-1$ binary responses. For instance, for $k=5$, binary responses are: 1 vs 2345 (recoded to 0 vs 1 ), 12 vs 345,123 vs 45,1234 vs 5 . Then it estimates two-parameter IRT item characteristic curves (ICC) for each $t$ binary recoded responses:

$$
P_{t}=\frac{1}{1+\exp \left(-\left(a_{i} \theta_{j}+c_{i t}\right)\right)}
$$


On this formulation $c_{i t}$ is the item difficulty of these recoded binary responses, that is, the point on the latent trait where there is a $50 \%$ chance of choosing 2345 vs 1,345 vs 12 and so on. This is also called between-categories threshold parameters. Note that they have to be ordered within items. The model constrains discrimination parameter $a_{i}$ to be equal across $k-1$ binary responses for the same item. Then the probabilities for each $k$ response categories is obtained by subtraction (Embretson \& Reise, 2000): $P\left(x=k \mid \theta_{j}\right)=P_{t}-P_{t-1}$ where $P\left(x=1 \mid \theta_{j}\right)=1-P_{2}$ and $P\left(x=5 \mid \theta_{j}\right)=P_{4}-0$.

$P_{t}$ can be expressed also as:

$$
\ln \left(\frac{P_{t}}{1-P_{t}}\right)=a_{i} \theta_{j}+c_{i t}
$$

The linear part $a_{i} \theta_{j}+c_{i t}$ can incorporate additional dimensions as: $a_{1 i} \theta_{1 j}+a_{2 i} \theta_{2 j}+$ $c_{i t}$. Therefore, the random intercept can be estimated as second factor $a_{2 i} \theta_{2 j}$ by fixing negative items' discriminations $a_{2 i}$ as -1 and positive items as +1 and estimating the variance of $\theta_{2 j}$. Note that this formulation requires reversing negative items before running the analysis. We can rewrite this equation as:

$$
\ln \left(\frac{P_{t}}{1-P_{t}}\right)=a_{i} \theta_{j}+z_{j}+c_{i t}
$$

With this setup, random intercept will be similar to the acquiescence index $z_{j} \simeq a c q_{j}$ and the latent trait is a DPF corrected measure similar to the scores corrected for acquiescence: $\theta_{j} \simeq$ scr. $r e c_{j}$. In order to make this relationship more salient let's consider two semantically opposite items, one PK item ip and another NK item in, such as, "I do my tasks the best way I can" vs "I put little effort in my tasks" where a subject should give a binary yes/no answer. The yes response is scored as $s=1$. Let's reverse the NK item computing $1-s_{\text {in }}$ so that its score will be in the 
same direction as the PK item $s_{i p}$. Assume also that both questions measure the same latent trait, have the same difficulty $b_{i p}=b_{i n}=b_{i}$, same discrimination $a_{i p}=a_{i n}=a_{i}$, and finally that the latent trait is not correlated with acquiescence $\left(r_{z \theta}=0\right.$ see justifications for this assumption in Ferrando et al. 2003). The log-odds of scoring 1 on these two items are, for the positively worded item:

$$
\ln \left(\frac{P_{s_{i p}}}{1-P_{s_{i p}}}\right)=a_{i} \theta_{j}+\left(1 z_{j}\right)+b_{i}
$$

And for the negatively worded item:

$$
\ln \left(\frac{P_{s_{i n}}}{1-P_{s_{i n}}}\right)=a_{i} \theta_{j}+\left(-1 z_{j}\right)+b_{i}
$$

If there is no DPF, subjects will answer both items in the same way but reflected. Since the negative item was reversed, the scored answers are expected to be the same. For a subject with a given $\theta_{j}$ the probability of scoring 1 will be equal in both items $P_{s_{i p}}=P_{s_{i n}}$. So the Item Characteristic Curves (ICC) will be the same. If there is DPF subjects no longer answer each item in the same way and the ICC's will differ. This is indeed a characteristic situation that defines DIF, that is, a given level on the primary trait having different probabilities of scoring 1 on the item. If random intercept $z_{j}$ is an index of DPF, it should capture these differences. If we subtract $P_{s_{i p}}-P_{s_{i n}}$ we see that this relationship holds true:

$$
\ln \left(\frac{P_{s_{i p}}}{1-P_{s_{i p}}}\right)-\ln \left(\frac{P_{s_{i n}}}{1-P_{s_{i n}}}\right)=a_{i} \theta_{j}+\left(+1 z_{j}\right)+b_{i}-\left[a_{i} \theta_{j}+\left(-1 z_{j}\right)+b_{i}\right]=2 z_{j}
$$

That is, for a subject with $z_{j}=0$, indicating no acquiescence, the ICC's will be equal (difference between probabilities will be equal to zero) indicating that they answered positive and negative items equally. If there is acquiescence, then $z_{j}>0 \Rightarrow P_{s_{i p}}>P_{s_{i n}}$, that is, it will be 
relatively easier to score 1 on the positive versus the negative item. In case of disacquiescence, $z_{j}<0 \Rightarrow P_{s_{i p}}<P_{s_{i n}}$, that is, the opposite pattern will occur, and it will be relatively easier to score 1 on the negative item (remember that the negative item was already reversed) ${ }^{4}$.

This relationship can be extended to Likert scales. The difference being that there will be one equation to each binary response with different item difficulties indexed by $c_{i t}$. When PK and NK items measure the same construct but have different parameters the relationship will be more complex given by:

$$
\begin{aligned}
& \ln \left(\frac{P_{s_{i p}}}{1-P_{s_{i p}}}\right)-\ln \left(\frac{P_{s_{i n}}}{1-P_{s_{i n}}}\right)=a_{i p} \theta_{j}+\left(+1 z_{j}\right)+b_{i p}-\left[a_{i n} \theta_{j}+\left(-1 z_{j}\right)+b_{i n}\right] \\
& \quad=\theta_{j}\left(a_{i p}-a_{i n}\right)+\left(b_{i p}-b_{i n}\right)+2 z_{j}
\end{aligned}
$$

${ }^{4}$ It is interesting to note the parallel of this model and the traditional way to solve DIF. In theory the cause of DIF is a secondary dimension that also influences the probability of answering, for instance, 1 , beyond the primary dimension. Subjects from two groups, A and B, that exhibit DIF in an item will have different latent scores on this secondary dimension. Therefore, considering all subjects with the same level on the primary dimension, subjects of group A will show different probabilities of answering 1 as compared to subjects of group B because of the secondary dimension. Procedures to resolve DIF create two test forms A and B where items that exhibit DIF are considered as unique to each form.

Consequently, instead of having only one item difficulty parameter, they will have two item difficulties, one for each group, as if they were different items. This is a form -by-group setup. Although DIF appears as an item effect, it really reflects a person effect as well because it is an interaction person by item. In the item response function it will appear as a constant value for item difficulty for DIF items, only for individuals of one of the groups, proportional to group difference. In this way individual differences in the secondary dimension that cause DIF will be modelled as an average difference for all subjects of a given group represented via different item difficulties. But we could also implement this procedure via random intercept. Once items that exhibit DIF are identified, we can setup a fixed set of weights proportional to DIF size in items that exhibit DIF and 0 on items with no DIF and estimate variance of this secondary factor. With this setup we will directly estimate individual differences in the secondary dimension that is producing DIF and will disentangled variance due to this secondary dimension from the primary dimension. Although DIF is caused by a "person" effect, both approaches differ in the way they model it. Either as an average effect equal for all persons of one group modeled as item difficulties or as an second dimension directly, that is, an continuous individual differences variable that affects only a subset of items. 
Therefore, more generally, differences in likelihood of scoring 1 in semantic opposite items are related to the differences in the item-trait relationship, $a_{i p}-a_{i n}$, differences in item difficulties $b_{i p}-b_{i n}$, and lastly the presence of DPF $2 z_{j}$. One important advantage of IRT modeling is that all item parameters are modeled simultaneously making it possible to identify DPF unconfounded with eventual global differences in PK and NK item characteristics.

\section{Research questions}

We have presented the conceptualization that Ten Berge's (1999) ipsatizing method is a way to solve DPF. We also noted this method is equivalent to a random intercept model for unidimensional balanced scales. Following this conceptual outline, these three methods to deal with acquiescence will now be applied and compared. We will first present a simulation study and subsequently an empirical example to expand our conceptual understanding of acquiescence correction of social- emotional skill scores using an IRT approach. Our goal is to demonstrate the relationships outlined in the introduction namely that (a) DPF calculated via an IRT method is equivalent to the acquiescence index $a c q_{j}$, (b) $a c q_{j}$ is equivalent to the random intercept $z_{j}$, and (c) recoded scores $s c r . r e c_{j}$ are equivalent to $\theta_{j}$ and are a solution to DPF, that is, high acquiescent individuals will have less extreme scores $\theta_{j}$ than non-acquiescent individuals given similar response patterns. Finally, in the simulation analyses, it will be also examined whether those relationships generalize to the case of unbalanced scales.

\section{Simulation Study}

\section{Goals}

The general purpose of the simulation study was to demonstrate the primary relationships among classical indices of acquiescence, DPF and an IRT version of a random intercept model, 
the relationships between the classical method of controlling for acquiescence and a modern version correcting via IRT modeling. In addition, we aimed to examine the generalizability of these relationship patterns to other conditions of unbalanced scales. Previous studies have noticed the relationship between acquiescence and DPF (Johanson, \& Osborn, 2004) and have modeled acquiescence with random intercept (Maydeu-Olivares, \& Coffman, 2006), but no study has connected acquiesce with DPF in the framework of IRT modeling.

\section{Method}

We started from a model of two uncorrelated factors representing trait and acquiesce variance. True trait and acquiescence factors were simulated from a normal distribution with a $M=0$ and $S D=1$ and $M=0$ and $S D=.40$ respectively. Samples of 10,000 subjects were generated for each replication. We generated three tests of 12-Likert scale items with five response categories according to Samejima's graded response model with the following features: (a) a balanced test having $6 \mathrm{PK}$ items and $6 \mathrm{NK}$ items (BL), (b) an unbalanced test towards the positive side having $9 \mathrm{PK}$ and $3 \mathrm{NK}$ items (Unbalanced Positive: UP), and (c) an unbalanced test towards the negative side having $3 \mathrm{PW}$ and $9 \mathrm{NW}$ items (Unbalanced Negative: UN). In each of these conditions 50 replications of 10.000 subjects were generated.

Item discrimination parameters for the trait factor were generated from a log normal distribution with $M=1.26$ and $S D=.42$. Item discrimination parameters for the acquiescence factor were fixed as +1 for PK and -1 for NK items. Item thresholds were randomly selected from a set of 30 item-calibrations ( $15 \mathrm{PK}$ and $15 \mathrm{NK})$ from our empirical example (see the next section). We selected PK and NK item thresholds from the subsets of $15 \mathrm{PK}$ and $15 \mathrm{NK}$ items respectively. The selected values of parameters defined above (SD for acquiescence M and SD 
for discrimination parameters and thresholds) were based on the values found on the empirical example so as to simulate a typical setup of large scale assessments of socio-emotional skills.

In total we had simulated 150 samples of 10,000 subjects ( $50 \times 3$ condition types of balancedness). For each sample we calculated several indices within each method studied: (a) classical scores method (Ten Berge, 1999): total average agreement scores, indices of acquiescence and scores corrected for acquiescence (this involves subtracting the mean acquiescence and was already described in the introduction); (b) DPF method (Linacre, 2017, Andrich, 1978, de Ayala, 2009, Linacre, 2017) in three steps, first estimating Samejima's graded response model parameters for persons and items in one calibration considering all items; secondly estimating again the person parameters twice: one using PK items $\theta_{p j}$ and the other using the NK items $\theta_{n j}$ while keeping items anchored at the item parameters from the main analysis; third calculating $P D I F_{j}=\theta_{n j}-\theta_{p j}$. If persons function in the same way while answering items they should have similar trait estimates. The difference should be within the confidence intervals computed from standard error of measurement. Therefore, the difference between these two scores indexes DPF; and finally, (c) Multidimensional Samejima's graded response model estimating random intercept (random intercept -RI method, Maydeu-Olivares \& Coffman, 2006). This was the two-factor model distinguishing trait and acquiescence used in the simulation. We estimated item thresholds and discrimination parameters for the trait factor, whereas for the acquiescence factor we fixed item discrimination parameters as +1 for the PK and -1 for NK items and estimated the factor's variance.

We further estimated scores of a unidimensional graded response model solution to compare wat happens when acquiescence (do-nothing approach examined by Savalei \& Falk 2014) is not modelled. An aditional random intercept model was also estimated changing the 
values of the discrimination parameters trying to compensate for unbalancedeness. We calculated

$a_{P k}=\left(n_{i t} / 2\right) / n_{p k}$ and $a_{n k}=\left(n_{i t} / 2\right) / n_{n k}$ where $n_{i t}$ is the total number of items and $n_{p k}$ and $n_{n k}$ were the number of PK and NK items respectively. Then we fixed PK item discrimination at $a_{P k}$ and NK at $a_{n k}$.

The primary variables of interest were the scores estimated with different methods (DPF trait scores and acquiescence scores) and their relationship. So we computed correlations among all scores and with true values of trait and acquiescence for each sample. We then averaged values across replications in the three balance-conditions (BL, UP, and UN).

All simulations and calculations were done in R language mirt package that can flexibly estimate multidimensional IRT models (Chalmers, 2012). We shared the R functions as well as additional data for those interested (https://github.com/rprimi/acqu_mirt).

\section{Results}

Table 2 presents the main results of the simulations. It presents the average correlations among several scores calculated from three different methods in three conditions (BL, UP and UN). The first three variables are scores from the classical method (average agreement, recoded scores and acquiescence index). Variables 4 is the DPF index. Variables 5 and 6 are trait and acquiescence scores estimated by the multidimensional graded response model with random intercept. Variables 7 and 8 (only shown in unbalanced conditions) are estimated with the same model but with different discrimination parameters trying to correct for unbalancedness. Variable 9 is a trait score estimated with a unidimensional model and variables 10 and 11 are the true trait and acquiescence scores from where the data were simulated. 
Table 2. Average descriptive statistics and correlations across 50 replications by conditions (BL,

UP and UN) among estimated measures via three models: classical scores, DPF, multidimensional IRT random intercept and true values.

\begin{tabular}{|c|c|c|c|c|c|c|c|c|c|c|c|c|}
\hline & $\mathrm{M}$ & $\mathrm{SD}$ & 1 & 2 & 3 & 4 & 5 & 6 & 7 & 8 & 9 & 10 \\
\hline & & & \multicolumn{10}{|c|}{ Balanced (BL) } \\
\hline Classical method & & & & & & & & & & & & \\
\hline 1. $s c r_{j}$ & 2.62 & 0.67 & & & & & & & & & & \\
\hline 2. $\operatorname{scr} r e c_{j}$ & 0.62 & 0.67 & 1.00 & & & & & & & & & \\
\hline 3. $a c q_{j}$ & 1.98 & 0.35 & -0.11 & -0.11 & & & & & & & & \\
\hline IRT DPF & & & & & & & & & & & & \\
\hline 4. $D P F_{j}$ & 0.0 & 0.76 & -0.03 & -0.03 & 0.96 & & & & & & & \\
\hline Multid. IRT Random Int & & & & & & & & & & & & \\
\hline 5. F1 trait $\theta_{j}$ & 0.0 & 0.92 & 0.98 & 0.98 & -0.08 & 0.00 & & & & & & \\
\hline 6. F2 RI $z_{j}$ & 0.0 & 0.47 & 0.00 & 0.00 & 0.98 & 0.98 & 0.03 & & & & & \\
\hline Unidimensional & & & & & & & & & & & & \\
\hline 9. F1 1d & 0.0 & 0.91 & 0.98 & 0.98 & -0.07 & 0.01 & 1.00 & 0.03 & 1.00 & 0.03 & & \\
\hline True person parameters & & & & & & & & & & & & \\
\hline 10. F1 true trait $\theta_{j}$ & 0.0 & 1.00 & 0.90 & 0.90 & -0.07 & 0.01 & 0.92 & 0.03 & 0.92 & 0.03 & 0.91 & \\
\hline 11. F2 true acqu. $z_{j}$ & 0.0 & 0.63 & -0.02 & -0.02 & 0.73 & 0.74 & 0.00 & 0.75 & 0.00 & 0.75 & 0.00 & 0.00 \\
\hline
\end{tabular}

Classical method

\section{Unbalanced positive (UP)}

1. $s c r_{j}$

2. scr.rec $_{j}$

3. $a c q_{j}$

IRT DPF
4. $D P F_{j}$

Multid. IRT Random Intercept

5. F1 trait $\theta_{j}$

6. F2 RI $z_{j}$

RI with modified $a$ weights

Unidimensional model
7. F1b trait $\theta_{j}$
8. F2b RI $z_{j}$
9. F1 1d

True person parameters

10. F1 true trait $\theta_{j}$

11. F2 true acqu $z_{j}$

Classical method
1. $s c r_{j}$
2. scr.rec $_{j}$

3. $\operatorname{acq}_{j}$

IRT DPF $\begin{array}{ll}2.58 & 0.67\end{array}$

$0.45 \quad 0.53$

0.95

$2.27 \quad 0.43$

$0.71 \quad 0.45$

$\begin{array}{lllll}0.0 & 0.76 & 0.44 & 0.14 & 0.92\end{array}$

$\begin{array}{llllll}0.0 & 0.91 & 0.97 & 0.96 & 0.60 & 0.32\end{array}$

$0.0 \quad 0.53$

$\begin{array}{lll}0.64 & 0.37 & 0.98\end{array}$

$0.95 \quad 0.53$

$\begin{array}{rrrrrrrrr}0.0 & 0.92 & 0.99 & 0.92 & 0.72 & 0.48 & 0.98 & 0.67 & \\ 0.0 & 0.33 & 0.14 & -0.19 & 0.78 & 0.93 & 0.00 & 0.84 & 0.17 \\ 0.0 & 0.92 & 0.98 & 0.88 & 0.78 & 0.55 & 0.96 & 0.73 & 1.00\end{array}$

$\begin{array}{ll}0.0 & 0.92\end{array}$

$0.98 \quad 0.88$

$\begin{array}{ll}0.78 & 0.55\end{array}$

$0.0 \quad 1.00$

$\begin{array}{ll}0.89 & 0.88\end{array}$

0.54

0.29

0.91

0.48

$\begin{array}{lll}0.90 & 0.00 & 0.88\end{array}$

$0.0 \quad 0.63$

$0.21-0.02$

0.62

Unbalanced negative (UN)
$\begin{array}{ll}2.63 & 0.69\end{array}$
$\begin{array}{lll}0.46 & 0.50 & 0.95\end{array}$
$\begin{array}{llll}1.66 & 0.49 & -0.81 & -0.58\end{array}$ 
$\begin{array}{llllll}\text { 4. } D P F_{j} & 0.00 & 0.78 & -0.52 & -0.23 & 0.90\end{array}$

Multid. IRT Random Intercept

5. F1 trait $\theta_{j}$

6. F2 RI $z_{j}$

RI with modified $a$ weights

Unidimensional model

7. F1b trait $\theta_{j}$

8. F2b RI $z_{j}$

9. F1 1d

True person parameters

10. F1 true trait $\theta_{j}$

11. F2 true acqu $z_{j}$ $\begin{array}{llllll}0.0 & 0.91 & 0.96 & 0.97 & -0.67 & -0.36\end{array}$

$\begin{array}{lllllll}0.0 & 0.52 & -0.65 & -0.38 & 0.96 & 0.97 & -0.49\end{array}$

First, we consider the classical method. Original scores $s c r_{j}$ calculated as an average agreement with the items are correlated 1 with recoded scores $s c r . r e c_{j}$, that is, in balanced scales the total scores are already implicitly corrected for acquiescence. In the case of unbalanced scales correlations $s c r_{j}$ and $s c r . r e c_{j}$, are slightly lower than one. Correlation of acquiescence $a c q_{j}$ and $s c r_{j} / s c r . r e c_{j}$ tended to be zero and in unbalanced conditions it correlates with scores $a c q_{j}$ positively in UP and negatively in $\mathrm{UN}(r=.71$ and $r=-.81$ respectively). Considering the correlations of $s c r . r e c_{j}$ and $a c q_{j}$ with true values of trait $\theta_{j}$ and acquiescence $z_{j}$ we see that in the balanced condition it achieves $r=.90$ and $r=.73$ respectively. In the unbalanced conditions true trait $\theta_{j}$ correlates with acquiescence $a c q_{j}(r=.54 /-.60)$ but true acquiescence $z_{j}$ tend not not correlate with $s c r_{j}$ and $s c r . r e c_{j}$. This suggests that the unbalanced condition biases acquiescence with trait information. Therefore, corrected scores will tend to be indirect biased as well. Recoded scores $s c r . r e c_{j}$ tend to be less disperse ( $\mathrm{SD}=.53 / .50$ for UP and UN vs $\mathrm{SD}=.67$ for BL) and slightly less correlated with the true trait $(r=.88)$ but still very close to the balanced condition.

Second, we consider the DPF index. As predicted, DPF is highly related with the acquiescence index $(r=.96, .92$ and .90 for BL, UP, UN respectively). It also shows the expected 
correlation with true acquiescence ( $r=.74 / .68 / .68$ for the three conditions). It is also slightly biased by trait information in unbalanced conditions.

Third, we consider the multidimensional graded response with random intercept. Again, relationships are consistent with those outlined in the introduction. Random intercept parameters $z_{j}$ are highly correlated with acquiescence $a c q_{j}(r=.98 / .98 / .96)$ and DPF $(r=.98 / .95 / .97)$.

Relationships of estimated trait and acquiescence with true values are particularly important since this third method is the same as the true model behind simulations. Estimated trait $\theta_{j}$ parameters correlated $r=.92 / .91 / .90$ with true values in all conditions, and random intercept $z_{j}$ correlated $r=.75 / .65 / .56$ with true values of acquiescence ${ }^{5}$. Regardless of the condition the random intercept method recovered true parameters similarly. Nevertheless, the correlation between estimated trait and random intercept seems to be biased positively in UP $(r=.53)$ and negatively in UN ( $r=-.49)$.

Finally, we consider two additional models (a) the multidimensional graded response model with random intercept with modified fixed weights and (b) the unidimensional model. For the modified weights we found similar results as the original model. One important difference is

${ }^{5}$ Since this is the correct model it is surprising that correlation of estimated acquiescence with true values doesn't achieve values close to 1 . One feature to note is that the information we have to estimate the random intercept is much lower than the trait. The proportion of variance explained by random intercept is generally around $7 \%$ as compared to $29 \%$ for the trait. Since this is related to the item's loading/discriminations, and discrimination is related to information - inverse of error of measurement - the error of measurement to estimate random intercept is large as compared to the one to estimate trait. Therefore, the magnitude of correlations of estimated values with true values obtained from the multidimensional graded response model with random intercept is the high benchmark possible given the error of measurement. It may be difficult to see the relative lower information of acquiescence factor since item discriminations parameters are all fixed to 1 or -1 . But because it explains lower amount of variance of item responses, to compensate for these high values of fixed item discrimination, the variance of latent factor of acquiescence is reduced (see a discussion in Baker 1990 on IRT parametrizations). 
that biased correlation among estimated trait and acquiescence largely reduced in the modified model $(r=.17 /-.14)$. The general factor of unidimensional solution, one that not models acquiescence, correlated .96-1.00 with the estimated trait factor showing equivalent results. But model fit indices of the unidimensional model were lower than the multidimensional version. An average fit indices across 150 simulated datasets for the unidimensional model were $M 2=204.3$, $p=0.02, R M S E A=.023, T L I=.893, C F I=.93$ as compared to $M 2=17.5, p=0.47, R M S E A$ $=.002, T L I=.99, C F I=.99$ for the multidimensional model.

In synthesis, the simulation study has demonstrated empirically the conceptual connections between acquiescence and DPF. It also showed that an adapted version of random intercept model in the framework of a multidimensional IRT can estimate acquiescence/DPF index and trait that is controlled for acquiescence effect. This is true for balanced scales where acquiescence is automatically corrected. In unbalanced scales we found that structural relationships between estimated trait and acquiescence were biased. Changing the weights of the random intercept trying to compensate for differences in the number of PK vs NK items, however, can help to reduce this bias.

\section{Empirical Example}

\section{Goals}

We present now the results of an analysis of empirical data where we applied the same three models to test the relationships demonstrated in the simulation study. We will examine whether DPF, $a c q_{j}$ and random intercept $z_{j}$ are equivalent, and whether recoded scores $s c r . r e c_{j}$ are equivalent to trait factor $\theta_{j}$ estimated in the multidimensional graded response model with a random intercept. 


\section{Method}

\section{Participants}

Participants were 40,649 students (55.1\% females) from 495 schools located in 232 cities in the State of São Paulo Brazil, aged from 11 to $18(\mathrm{M}=14.7, \mathrm{SD}=1.99)$ attending grades $6^{\text {th }}$ $\mathrm{N}=6,411,7^{\text {th }} \mathrm{N}=4,357,8^{\text {th }} \mathrm{N}=5,558,9^{\text {th }} \mathrm{N}=6,272,10^{\text {th }} \mathrm{N}=6,534,11^{\text {th }} \mathrm{N}=5,763$ and $12^{\text {th }} \mathrm{N}=5,754$. All participated in social-emotional skill research conducted by Edulab21 from the Ayrton Senna Institute. The sample was relatively diverse, including many students from lower socioeconomic status backgrounds, more approximating school samples in Brazil than convenience samples of college students or web volunteers like in many studies in psychology. We report two student's proxy variables for socioeconomic status: (a) mother educational attainment: $29.8 \%$ of the mothers have not completed elementary grade, $23.6 \%$ with completed elementary grade, $34.3 \%$ completed high school, 12.3\% completed graduate school; (b) indirect measures of family income: $27 \%$ of the students came from families eligible for federal program of family scholarship and about $14.6 \%$ lived in houses where there wasn't sidewalk.

\section{Measures}

Students provided self-ratings on SENNA v2.0. (Primi, Santos, De Fruyt, \& John, in press), but the analyses reported here are constrained to the Conscientious Self-management domain. This scale is composed of five facets measuring Achievement, Concentration, Order, Self-discipline and Sense of Responsibility. Each facet is assessed by six items: three true keyed (positive items, for instance "I do my tasks the best way I can") and three false keyed (negative items, for instance "I put little effort in my tasks"), forming a fully balanced 30-item scale with 15 pairs of antonym items. Items were answered on a 5-point Likert scale: '1' (not at all like me), '2' (little like me), '3' (moderately like me), '4' (a lot like me) and '5' (completely like me). 


\section{Design and Data Analysis}

We calculated scores for the same three models described in the simulation study: classical scores' method, DPF method and Multidimensional Samejima's graded response model estimating random intercept. We used weights +1 and -1 for estimating random intercepts since the scale was balanced. Data and code are available at (https://github.com/rprimi/acqu_mirt).

\section{Results}

Table 3 shows descriptive statistics and correlations among the main parameters estimated under the three models. Results are very similar to those found in the simulation study. As predicted, DPF, acquiescence $a c q_{j}$ and random intercept parameters $z_{j}$ correlate very highly ( $r$ form .96 to .98 ). Substantive trait $\theta_{j}$ correlated very high with $s c r_{j}$ and $s c r . r e c_{j}(r=.97)$.

Table 3. Descriptive statistics and correlations among estimated measures via three models: Ipsatization, IRT DPF, and random intercept

\begin{tabular}{|c|c|c|c|c|c|c|c|}
\hline Variable & $M$ & $S D$ & 1 & 2 & 3 & 4 & 5 \\
\hline \multicolumn{8}{|l|}{ Ipsatization } \\
\hline 1. Original: $s c r_{j}$ & 3.58 & 0.58 & & & & & \\
\hline 2. Recoded: scr. $r e c_{j}$ & 0.58 & 0.58 & $1.00 * *$ & & & & \\
\hline 3. Acquiescence: $a c q_{j}$ & 2.97 & 0.35 & $-.04 * *$ & $-.04 * *$ & & & \\
\hline \multicolumn{8}{|l|}{ IRT } \\
\hline 4. $D P F_{j}$ & 0.03 & 0.96 & $-.08 * *$ & $-.08 * *$ & $.96 * *$ & & \\
\hline \multicolumn{8}{|l|}{ Random intercept } \\
\hline 5. F1 (Trait, $\theta_{j}$ ) & -0.01 & 0.96 & $.97 * *$ & $.97 * *$ & $.05 * *$ & .00 & \\
\hline 6. F2 (RI, $\left.z_{j}\right)$ & -0.00 & 0.56 & $-.08 * *$ & $-.08 * *$ & $.98 * *$ & $.98 * *$ & .01 \\
\hline
\end{tabular}

Note. ${ }^{*} p<.05 ; * * p<.01 . M=$ mean; $S D=$ standard deviation. F1 (trait) is the trait factor and F2 (RI) is the random intercept. 
The upper part of Figure 2 shows the relationship between the recoded scores $s c r . r e c_{j}$ on the y-axis and the acquiescence index $a c q_{j}$ on the x-axis. It also shows DPF measures as shades of gray. This figure clearly illustrates how DPF relates to recoded scores. If acquiescence $a c q_{j}$ is close to the expected score of 3 (that is $\mathrm{DPF}=0$ ) recoded scores span to a full amplitude of -2 to 2 . The more the acquiescence index goes to extremes (towards 5 indicating high acquiescence or towards 1 indicating disacquiescence) recoded scores become less extreme reducing the amplitude of variation. The lower part of Figure 2 shows the equivalent parameters but now obtained in random intercept model. It shows a parallel pattern since $P D I F \simeq z_{j} \simeq$ $a c q_{j}$ and the latent trait $\theta_{j} \simeq s c r . r e c_{j}$. These results are consistent with the idea that a multidimensional graded response model with random intercept produces measures corrected for DPF. Both methods make scores less extreme as a function of individual differences on $\mathrm{DPF} /$ acquiescence index.

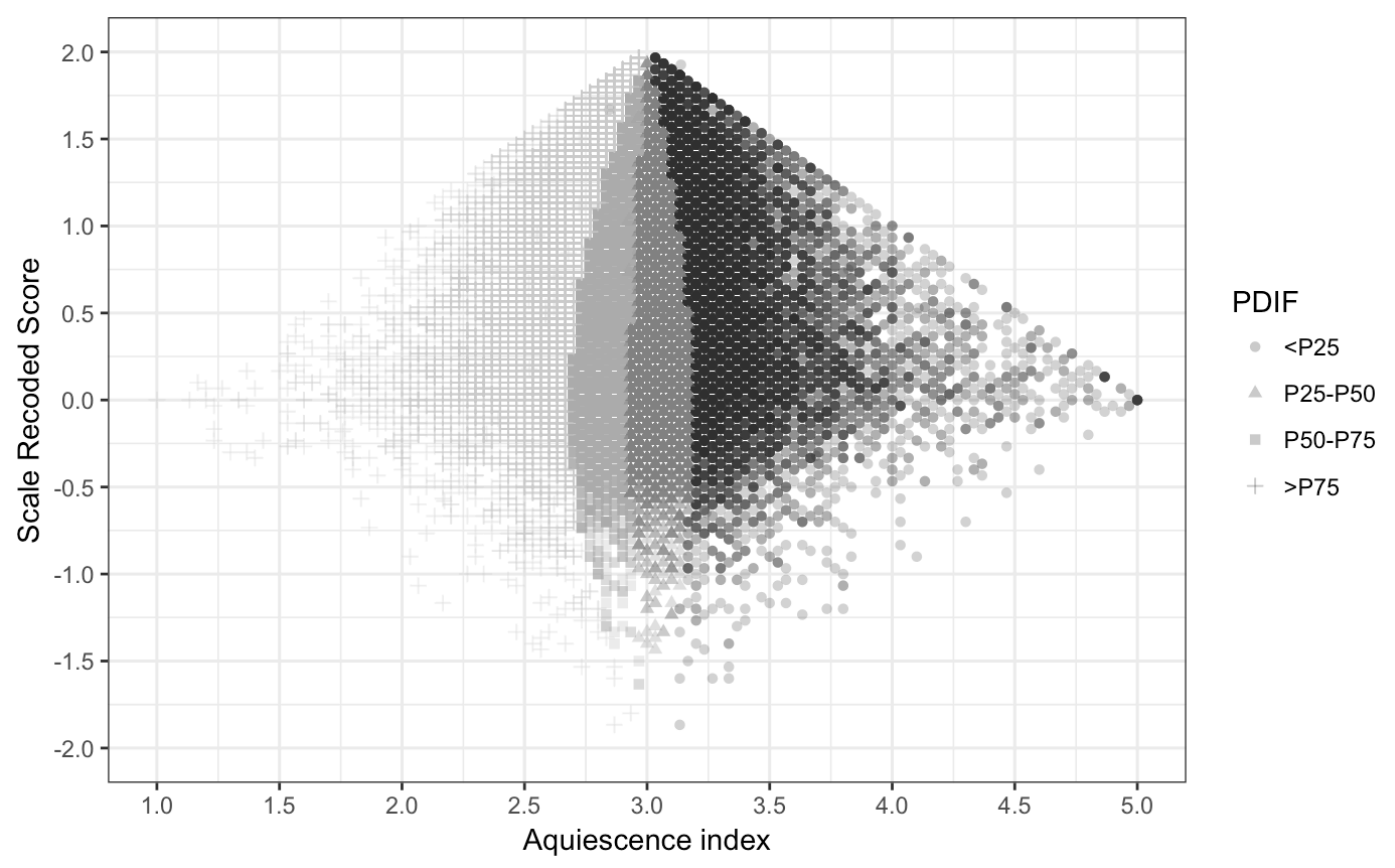




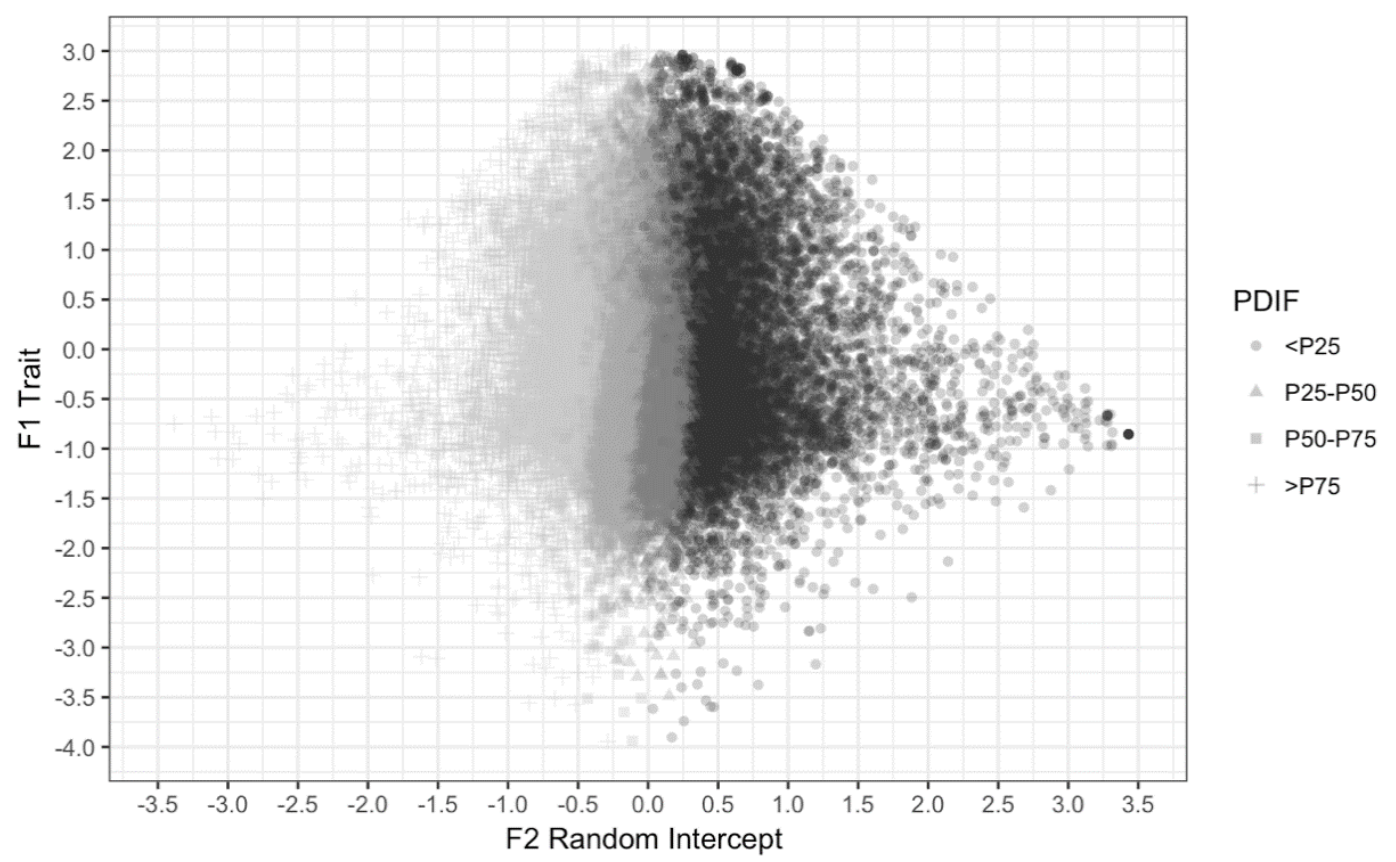

Figure 2. Relationship between recoded scores $s c r . r e c_{j}$ (y-axis) and acquiescence index $a c q_{j}$ (x-axis): - upper and random intercept substantive trait $\mathrm{F} 1\left(\theta_{j}\right)$ random intercept $\mathrm{F} 2\left(z_{j}\right)$. colored by the level in DPF - lower figure.

\section{Discussion}

Self-report questionnaires rely on the assumption that their items reflect information only about the traits they are supposed to measure; that is, persons will respond mainly to the content of the questions. The item response process, however, is complex and multidimensional, and involves other factors that may substantially affect subjects' responses (McCrae, 2919, Wetzel \& Carstensen, 2015). Especially in children, the response style of acquiescence is an important source of systematic error (Soto et al., 2008). Balanced scales have been proposed as a solution to control for acquiescence (Jackson, 1971), but the reason why they work has not been fully explicated using modern psychometric models.

We began with Johanson and Osborn's (2004) proposal that acquiescence can be understood in IRT as DPF, that is, the process of responding differently to items measuring the 
same trait with equivalent difficulty but with different item features. We elaborated DPF in the context of a multidimensional IRT model as an extra factor beyond substantive trait variance. The present results from both simulations and empirical data are strongly convergent and showed that: (a) the acquiescence index can be regarded as very similar to DPF, (b) balanced scales resolve the problems posed by DPF because in balanced scales acquiescence is partialled out, and (c) the random intercept can be regarded as very similar to both the acquiescence index and DPF, while the substantive trait factor is controlled for DPF.

To understand the functioning of balanced scales, two item features are of crucial importance: (a) behavior of agreement and (b) behavior in the direction of item keying. First, the average endorsement on the rating scale (before reversing any scores) will capture how much the individual agreed on all the items. Second, keying direction involves whether the item content is phrased in the direction of the high pole of the construct (e.g. extraversion) or the low pole of the construct (e.g. introversion). In true or positively keyed items, a high position on the latent trait is associated with agreement, whereas in false or negatively keyed items this position is associated with disagreement. Summing these items after reversing scores on negatively keyed items will capture how high the person is scoring on the underlying trait. Therefore, the process of responding to the substantive content of the item will be captured by the consistency of reflected responses on antonym pairs, that is, by the behavior in the direction of the keying. The bias component in the response process (e.g., difficulty or ambiguity of understanding the item) is captured by the acquiescence score, that is, overall behavior of agreement (or disagreement in the case of disacquiescence) with antonym pairs (see Primi et al., 2018).

These concepts of item design that try to purify measurement manipulating item features have been commonly used in cognitive measurement (De Boeck, \& Wilson, 2004; Embretson, 
1983, 1994; Primi, 2014) but only rarely in personality assessment. A thoughtful example of item design was presented by Mirowsky and Ross (1991) who analyzed internal and external locus of control with items that allowed them to isolate acquiescence and defensiveness. They used the same principle of balancing item features with difference scores between groups of items to identify substantive information separately from bias.

The combination of item design used here, and the random intercept measurement model makes it possible to frame the old approach of partialling out acquiescence bias within the new approach using multidimensional IRT models. Conceptually, random intercept models represent DPF as item difficulty parameters. They split item difficulty into two components (MaydeuOlivares \& Coffman, 2006): (a) $c_{i}$ main effect of item difficulty accounting for how globally easy it is to agree with items regardless of who is answering (subscript indicates that it will only vary over items $i$ ) and (b) $z_{j}$, a person main effect of how easy it is to agree with all items regardless of their content. Since $z_{j}$ is a random effect and varies over $j$ persons, it is equivalent to a second latent factor. We showed that this parameter could be interpreted as a person-tailored adjustment of item difficulty based on their tendency to agree indiscriminately. A person A with the same trait level as person $\mathrm{B}$ on substantive trait $\theta_{j}$, but manifesting high acquiescence $z_{A}>$ $z_{B}$, will have an inflated trait score if not adjusted down proportional to the difference in acquiescence. Therefore $z_{j}$ indexes how globally easy it is to agree (or disagree) for a particular person $j$ varying over all persons and adjusting test difficulty individually, partialling out this difference from substantive trait variance that becomes less influenced by acquiescence.

We showed that acquiescence correction happens automatically in balanced scales. But what happens when scales are unbalanced? Our simulations showed that one of the most important consequences of imbalance is a biased correlation between trait and acquiescence. 
Even if in the true model there is no correlation between the two factors, having more PK items produced a biased positive correlation and having more NK items produced a biased negative correlation between trait and acquiescence. This can potentially bias correlations with external criteria (see a detailed discussion in Mirowsky \& Ross 1991, Ferrando, et al. 2003; Rammstedt, at al. 2010). Consider for example an unbalanced scale towards PK items. If substantive trait variance is positively correlated with criteria and acquiescence is negatively correlated with criteria, since acquiescence will tend to correlate positively with the trait, it will act as a suppressor lowering the trait-criterion validity relationship. In a study of criterion validity of socio-emotional skills comparing only PK item scales versus only NK items scales evidence of this suppressor effect was found (Primi et al., 2018) ${ }^{6}$.

Our simulations also showed that employing a modified set of weights in the estimation of random intercept, to compensate for an imbalance of $\mathrm{PK} / \mathrm{NK}$ items, reduced the biased correlations between acquiescence and trait variance close to the true value of zero. This method could be used in most practical situations that use unbalanced scales.

The adaptation of the random intercept model implemented here was a simplified version to compare it directly with the classical method of partialling out the overall response mean (representing acquiescence). This simple model has some limitations. For instance, item discriminations (or item factor loadings) are modeled only on the substantive trait, but not on the acquiescence factor, since the random intercept is fixed to unit discrimination. Ten Berge (1999)

\footnotetext{
${ }^{6}$ It is interesting to note that imbalance will also occur if we have the same number of PK and NK items if they have different item discriminations (Savalei \& Falk, 2014).
} 
argues that items may be differentially influenced by acquiescence requiring different item loadings/discriminations.

In concluding, the present work made clear that total scores derived from fully balanced scales are automatically corrected for acquiescence. Therefore, the classical method is a simpler version of the DPF correction. Nonetheless, when we frame this process of correction explicitly within an IRT model, we can go beyond the simple assumptions of the classical model, allowing us to formulate and test more flexible types of models. These models can be estimated by a generalized multidimensional IRT model developed by Falk and Cai (2016) that provides greater power and complexity than the classical approach. The present paper has systematically examined different psychometric ways to deal with acquiescence, how these are related, and it has further increased insight into how such corrections work. Large-scale social-emotional skill assessment via self-reports should benefit from these methods and insights.

\section{References}

Abrahams, L., Pancorbo Valdivia, G., Primi, R., Santos, D., Kyllonen, P., John, O., \& De Fruyt, F. (in press). Social-emotional skill assessment in children and adolescents: Advances and challenges in personality, clinical, and educational contexts. Psychological Assessment

Baker, F. B. (1990). Some Observations on the Metric of PC-BILOG Results. Applied Psychological Measurement, 14(2), 139150.https://doi.org/10.1177/014662169001400203

Andrich, D. (1978). A Rating Formulation for Ordered Response Categories. Psychometrika, 43(4), 561-573.

Chalmers, R. P. (2012). mirt: A Multidimensional Item Response Theory Package for the R Environment. Journal of Statistical Software, 48(6), 1-29. 
Chan, W., \& Bentler, P. M. (1998). Covariance structure analysis of ordinal ipsative data. Psychometrika, 63(4), 369-399. https://doi.org/10.1007/BF02294861

de Ayala, R. J. (2009). The theory and practice of item response theory. New York, NY, US: Guilford Press.

De Boeck, P., \& Wilson, M. (Eds.). (2004). Explanatory Item Response Models: A Generalized Linear and Nonlinear Approach. New York: Springer-Verlag.

Duckworth, A. L., \& Yeager, D. S. (2015). Measurement Matters. Educational Researcher. https://doi.org/10.3102/0013189X15584327

Embretson, S. E. (1983). Construct validity: Construct representation versus nomothetic span. Psychological Bulletin, 93, 179-197. https://doi.org/10.1037/ 0033-2909.93.1.179

Embretson, S. E. (1994). Applications of Cognitive Design Systems to Test Development. In: (eds) . In C. R. Reynolds., Cognitive Assessment. Perspectives on Individual Differences (p. 107:135). Boston, MA: Springer.

Embretson, S. E., \& Reise, S. (2000). Item Response Theory for Psychologists. Mahwah, NJ: Lawrence Erlbaum Associates.

Falk, C. F., \& Cai, L. (2016). A flexible full-information approach to the modeling of response styles. Psychological Methods, 21(3), 328-347. https://doi.org/10.1037/met0000059

Ferrando, P. J., Lorenzo-Seva, U., \& Chico, E. (2003). Unrestricted Factor Analytic Procedures for Assessing Acquiescent Responding in Balanced, Theoretically Unidimensional Personality Scales. Multivariate Behavioral Research, 38(3), 353-374. https://doi.org/10.1207/S15327906MBR3803_04

Garrido, L. E., Golino, H., Nieto, M. D., Peña, K. G.\& Molina, A. M. (2018). A Systematic Evaluation of Wording Effects Modeling Under the ESEM Framework. Paper presented 
at the International Meeting of the Psychometric Society (IMPS), New York, NY.

He, J., Bartram, D., Inceoglu, I., \& van de Vijver, F. J. R. (2014). Response Styles and Personality Traits: A Multilevel Analysis. Journal of Cross-Cultural Psychology, 45(7), 1028-1045. https://doi.org/10.1177/0022022114534773

Jackson, D. N. (1971). The dynamics of structured personality tests: 1971. Psychological Review, 78, 229-248.

Johanson, G. A., \& Osborn, C. J. (2004). Acquiescence as differential person functioning. Assessment \& Evaluation in Higher Education, 29(5), 535-548. https://doi.org/10.1080/02602930410001689126

Kamata, A., \& Bauer, D. J. (2008). A note on the relation between factor analytic and item response theory models. Structural Equation Modeling, 15(1), 136-153. https://doi.org/10.1080/10705510701758406

Kyllonen, P. C., Lipnevich, A. A., Burrus, J., \& Roberts, R. D. (2014). Personality, Motivation, and College Readiness: A Prospectus for Assessment and Development: Personality, Motivation, and College Readiness. ETS Research Report Series, 2014(1), 1-48. https://doi.org/10.1002/ets2.12004.

Linacre, J. M. (2017). Winsteps Rasch measurement computer program User's Guide. Oregon: Winsteps.com. Retrieved from http://www.winsteps.com/winman/

McCrae, R. R. (2018). Method Biases in Single-Source Personality Assessments. Psychological Assessment. Advance online publication. http://dx.doi.org/10.1037/pas0000566

Maydeu-Olivares, A., \& Coffman, D. L. (2006). Random intercept item factor analysis. Psychological Methods, 11(4), 344-362. https://doi.org/10.1037/1082-989X.11.4.344

Mirowsky, J., \& Ross, C. E. (1991). Eliminating Defense and Agreement Bias from Measures of 
the Sense of Control: A 2 X 2 Index. Social Psychology Quarterly, 54(2), 127-145. https://doi.org/10.2307/2786931

Primi, R. (2014). Developing a fluid intelligence scale through a combination of Rasch modeling and cognitive psychology. Psychological Assessment, 26(3), 774.

Primi, R., Fruyt, F. D., John, O. P., \& Santos, D. (2018). Validities of True and False Keyed Social-emotional Skill Items Before and After Acquiescence Correction to Predict Educational Achievement. Paper submitted for publication.

Primi, R., Santos, D., De Fruyt, F., \& John, O. P. (in press). SENNA V2.0 Technical Manual. São Paulo: Instituto Ayrton Senna (IAS).

Rammstedt, B., Goldberg, L. R., \& Borg, I. (2010). The measurement equivalence of Big-Five factor markers for persons with different levels of education. Journal of Research in Personality, 44(1), 53-61. https://doi.org/10.1016/j.jrp.2009.10.005.

Samejima, F. (1968). Estimation of Latent Ability Using a Response Pattern of Graded Scores. ETS Research Bulletin Series, 1968(1), 1-169. https://doi.org/10.1002/j.23338504.1968.tb00153.x

Savalei, V., \& Falk, C. F. (2014). Recovering Substantive Factor Loadings in the Presence of Acquiescence Bias: A Comparison of Three Approaches. Multivariate Behavioral Research, 49(5), 407-424. https://doi.org/10.1080/00273171.2014.931800

Soto, C. J., \& John, O. P. (2017). The Next Big Five Inventory (BFI-2): Developing and Assessing a Hierarchical Model With 15 Facets to Enhance Bandwidth, Fidelity, and Predictive Power. Journal of Personality and Social Psychology, 113(1):117-143, http:// dx.doi.org/10.1037/pspp0000096

Soto, C. J., John, O. P., Gosling, S. D., \& Potter, J. (2008). The developmental psychometrics of 
big five self-reports: acquiescence, factor structure, coherence, and differentiation from ages 10 to 20. Journal of Personality and Social Psychology, 94(4), 718-737. https://doi.org/10.1037/0022-3514.94.4.718

Takane, Y., \& Leeuw, J. de. (1987). On the relationship between item response theory and factor analysis of discretized variables. Psychometrika, 52(3), 393-408. https://doi.org/10.1007/BF02294363

Ten Berge, J. M. (1999). A Legitimate Case of Component Analysis of Ipsative Measures, and Partialling the Mean as an Alternative to Ipsatization. Multivariate Behavioral Research, 34(1), 89-102. https://doi.org/10.1207/s15327906mbr3401_4

Wetzel, E., \& Carstensen, C. H. (2015). Multidimensional Modeling of Traits and Response Styles. European Journal of Psychological Assessment, 1-13. https://doi.org/10.1027/1015-5759/a000291

Wetzel, E., Lüdtke, O., Zettler, I., \& Böhnke, J. R. (2016). The Stability of Extreme Response Style and Acquiescence Over 8 Years. Assessment, 23(3), 279-291. https://doi.org/10.1177/1073191115583714 